\title{
Optical Interference and Self-Scattering Effect On Laser Ablation of Thin Silicon Films

\author{
Hao Zhang ${ }^{1}$, D. van Oosten ${ }^{1}$, D. M. Krol ${ }^{1,2}$, and J. I. Dijkhuis ${ }^{1}$ \\ ${ }^{I}$ Debye Institute for Nanomaterials Science, Utrecht University, \\ P. O. Box 80000, 3508 TA Utrecht, The Netherlands. \\ ${ }^{2}$ Department of Chemical Engineering and Materials Science, University of California, Davis. \\ h.zhang1@uu.nl
}

\begin{abstract}
We report measurement of the ablation depth and self-reflectivity from thin silicon films illuminated by a single tightly focused femtosecond laser pulse. We show the dependence of ablation depth on incident laser pulse fluence can be modeled with a transfer-matrix method, taking into account transient reflectivity and light propagation in a stratified medium. We find optical interference effects in laser ablation of thin films are of crucial importance. Furthermore, We present the evidence of self-scattering effects due to the buildup of a three dimensional submicron sized plasma in the focal region.
\end{abstract}

\section{Introduction}

The use of femtosecond lasers as a tool for precise machining of micron and submicron features in both dielectrics [1-2] and semiconductors [3] has been steadily growing in recent years. Thin film silicon such as the silicon-on-insulator (SOI) wafer has found wide application in silicon photonics and MEMS fabrication. Fast prototyping of photonic waveguide devices on SOI wafers by direct fs laser ablation has been reported [3]. For the bulk silicon case, several groups have utilized atomic force microscopy (AFM) to study the single shot ablation depth [4] as well as changes in self-reflectivity due to the excitation of carriers during the ablation process [5]. We use the term self-reflectivity to imply that the incident pulse creates a dense electron-hole plasma in the focal region thus significantly altering the reflectivity the laser pulse experiences. In our previous study, we performed AFM measurements of the single shot ablation depth in SOI wafers and identified three modification regimes [6]. In this study, we continue the work reported in [6] but did the experiments of single shot ablation depth and the self-reflectivity changes with an upgraded fully automated experimental setup. The experimental results as well as theoretical calculations emphasize the importance of the optical interference inside the thin film layer and the effect of self-induced scattering due to the buildup of a 3D submicron sized plasma in the focal region of the objective.

\section{Experiment setup}

The new setup is based on the basic fs laser ablation setup described in [6] but with many additional features to improve the precision and the reproducibility of the experiments. These new features are:

1. Measurement of the incident pulse energy at the single-shot level using the integrated signal from a photodiode. This allows us to measure the pulse energy individually and thus eliminate errors due to the shot-to-shot instabilities.

2. Determination of focused spot size for each individual laser shot in the experiment. This allows us to automatically and reproducibly focus the objective and to correct for fluctuations in focused laser fluence due to mechanical vibrations.

3. Automated acquisition and fitting of each reflected focal spot on the CCD camera. This allow us to measure the self-reflectivity changes as a function of incident fluence.

4 Automated fitting of the AFM data of the ablated craters. This allows us to quickly determine the single shot ablation depth of hundreds of craters.

\section{Results and discussion}

The experiments were done on 3 samples, bulk silicon and two SOI wafers with $217 \mathrm{~nm}$ and $120 \mathrm{~nm}$ device layers, respectively. The $217 \mathrm{~nm}$ SOI film had a $1 \mu \mathrm{m}$ thick insulating silica layer while the $120 \mathrm{~nm}$ SOI film had a $300 \mathrm{~nm}$ silica layer. Fig.1 shows the changes in single shot ablation depth and self-reflectivity as a function of incident pulse fluence. The general features of the ablation depth data for bulk silicon reveal two ablation regimes where the ablation depths are limited by the nonlinear optical penetration depth and the diffusive process respectively. We note that this finding is consistent with the results published by other groups [4]. The ablation 
results for the $217 \mathrm{~nm}$ film show a sudden step in the single shot ablation depth curve (Fig.1 (a)). The general trend of the self-reflectivity curves in Fig.1 (b) suggests a Drude response of the excited dense electron-hole plasma [5]. The differences in the data for the three samples immediately implies the importance of layer thickness on both the ablation and self-reflectivity behavior.
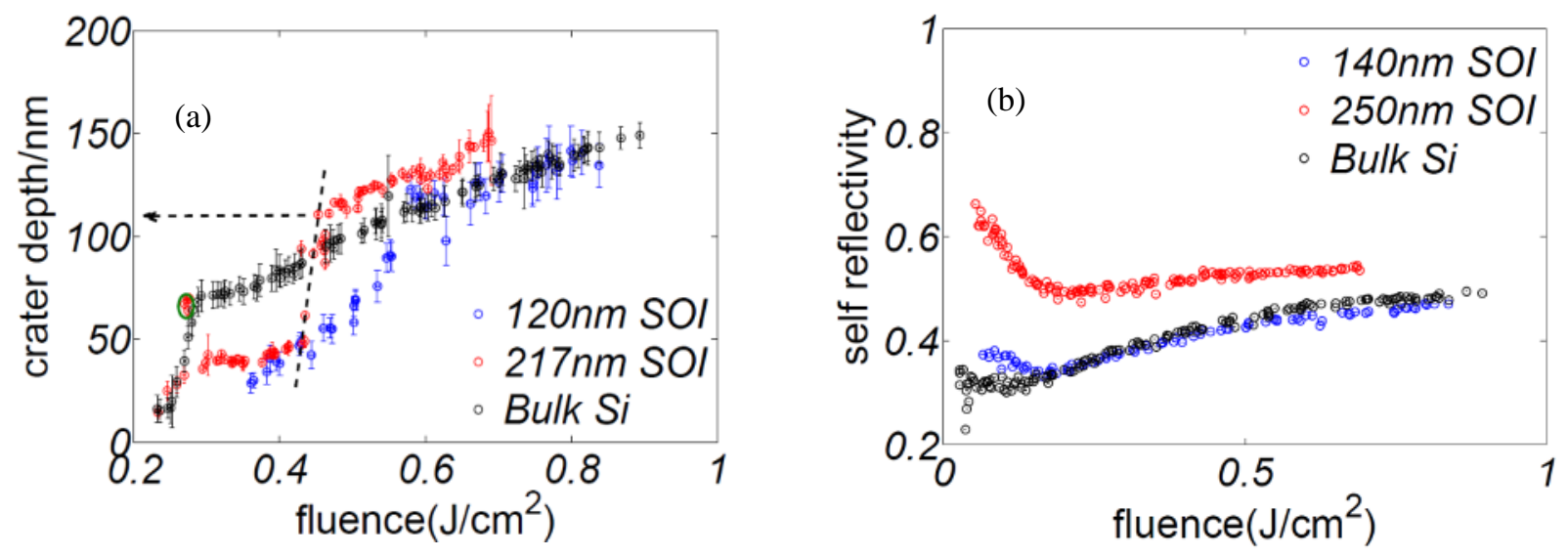

Fig. 1. (a) Single shot ablation depth. (b) Self-reflectivity.

Theoretical calculations were conducted using a 1D transfer matrix model which takes into account the layered structure of the SOI wafers and the transient change of dielectric constant in a stratified medium due to the excitation of a dense electron-hole plasma. Because of the high contrast in the refractive index between the silicon layer and the oxide layer beneath, as the laser pulse goes into the material, an interference pattern between the ingoing and outgoing light waves is established well before the pulse ends. As a result, a standing wave is formed inside the device layer. This standing wave redistributes the electro-magnetic energy in the film, giving rise to local minima and maxima in electric field strength, thus altering both the ablation depth and the self-reflectivity. The calculation for the $217 \mathrm{~nm}$ SOI film predicts a local minimum in the internal laser fluence at $\sim 50 \mathrm{~nm}$ and a local maximum in the internal laser fluence at $\sim 120 \mathrm{~nm}$, nicely consistent with the depth of the suppression and the sudden rise in ablation depth in Fig.1 (a). As a contrast, for the bulk silicon and the $120 \mathrm{~nm}$ SOI wafer, the model predicts much smaller contrast in the value of the local minimum and maximum, in fact not enough to show the interference effect. This is again consistent with the experimental data.

For a quantitative explanation of the self-reflectivity data plotted in Fig.1 (b), we are in the process of developing a 2D and 3D finite difference time domain method that incorporates the transient change of the dielectric constant due to the excitation of a dense electron-hole plasma. The FDTD simulation shows that a submicron sized plasma is created in the focal region and this plasma significantly influences the self-reflectivity. In the experiment, a 3D plasma is created in the focal region of the objective, where in our case the size of the plasma region is diffraction limited. The tiny plasma scatters the incident wave in all directions and therefore we propose that the term self-scattering is more appropriate than self-reflectivity in this case.

\section{References}

[1] "Femtosecond laser micromachining: photonic and microfluidic devices in transparent materials", Springer Series, Topics in Applied Physics, Vol. 123, Eds. R. Osellame, G. Cerullo, R. Ramponi, 2012.

[2] R. Gattass and E. Mazur, "Femtosecond laser micromachining in transparent materials," Nature Photonics 2, 219-225 (2008).

[3]Ming Li, Kiyotaka Mori, Makoto Ishizuka, Xinbing Liu, Yoshimasa Sugimoto, Naoki ikeda, and Kiyoshi Asakawa, " Photonic bandpass filter for $1550 \mathrm{~nm}$ fabricated by femtosecond direct laser ablation", Appl. Phys. Lett. 83, 216(2003).

[4]David J. Hwang, P. Grigoropoulos and Tae Y. Choi, " Efficiency of silicon micromachining by femtosecond laser pulses Efficiency of silicon micromachining by femtosecond laser pulses in ambient air", J. Appl. Phys 99, 083101 (2006).

[5]D. Hulin, M. Combescot, J. Bok, A.Migus, J. Y. Vinet and A. Antonetti,"Energy transfer during silicon irradiation by femtosecond laser pulse", Phy. Rev. Lett. 52, 1998 (1984)

[6]Hao Zhang, D.van Oosten, D. M. Krol, and J. I. Dijkhuis," Saturation effects in femtosecond laser ablation of silicon-oninsulator", Appl. Phys. Lett. 99, 231108 (2011). 